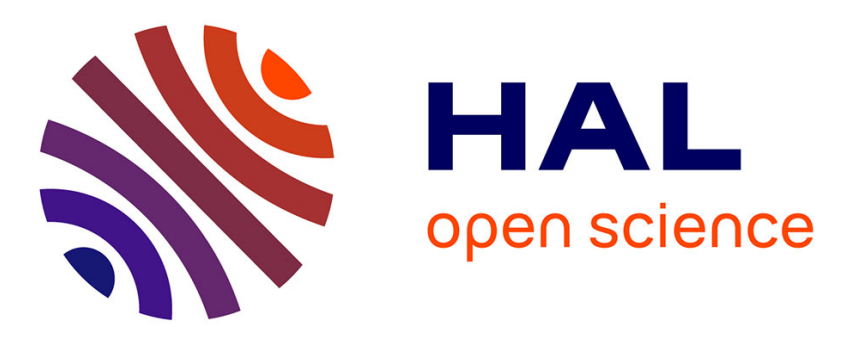

\title{
Damage localization of a real structure using the statistical subspace damage localization method
}

Saeid Allahdadian, Michael Döhler, Carlos Ventura, Laurent Mevel

\section{To cite this version:}

Saeid Allahdadian, Michael Döhler, Carlos Ventura, Laurent Mevel. Damage localization of a real structure using the statistical subspace damage localization method. IWSHM - 11th International Workshop on Structural Health Monitoring, Sep 2017, Stanford, United States. 10.12783/shm2017/14136 . hal-01589549

\section{HAL Id: hal-01589549 \\ https://hal.inria.fr/hal-01589549}

Submitted on 18 Sep 2017

HAL is a multi-disciplinary open access archive for the deposit and dissemination of scientific research documents, whether they are published or not. The documents may come from teaching and research institutions in France or abroad, or from public or private research centers.
L'archive ouverte pluridisciplinaire HAL, est destinée au dépôt et à la diffusion de documents scientifiques de niveau recherche, publiés ou non, émanant des établissements d'enseignement et de recherche français ou étrangers, des laboratoires publics ou privés. 


\section{COVER SHEET}

NOTE:

- Please attach the signed copyright release form at the end of your paper and upload as a single 'pdf' file

- This coversheet is intended for you to list your article title and author(s) name only

- This page will not appear in the book or on the CD-ROM

Title: Damage localization of a real structure using the statistical subspace damage localization method

Authors (names are for example only): Saeid Allahdadian ${ }^{1}$

Michael Döhler ${ }^{2,3}$

Carlos Ventura ${ }^{1}$

Laurent $\mathrm{Mevel}^{2,3}$

**IMPORTANT** All authors' information will appear on the program according to the submission stub on the online submission system (not to the manuscript). The title and author list provided in the manuscript will be for future referencing and citation.

PAPER DEADLINE: **May 15, 2017**

PAPER LENGTH: **8 PAGES MAXIMUM **

Please submit your paper in PDF format. We encourage you to read attached Guidelines prior to preparing your paper-this will ensure your paper is consistent with the format of the articles in the CD-ROM.

NOTE: Sample guidelines are shown with the correct margins.

Follow the style from these guidelines for your page format.

Electronic file submission: When making your final PDF for submission make sure the box at "Printed Optimized PDF" is checked. Also-in Distiller-make certain all fonts are embedded in the document before making the final PDF. 


\begin{abstract}
In this paper the statistical subspace damage localization (SSDL) method is employed in localizing the damage in a real structure, namely the Yellow frame. The SSDL method is developed for real testing conditions and tested in two damage configurations. It was demonstrated that the SSDL method can localize the damage robustly in the Yellow frame for simple and multiple distinct damage scenarios using the analytical modal parameters. The method is described and its effectiveness is demonstrated.
\end{abstract}

\title{
INTRODUCTION
}

With the advent of new technologies, instrumentation of structures is becoming widespread. The data acquired from the instrumented structures can provide beneficial information on their structural conditions. However, efficient techniques and methods are necessary in processing this data in order to assess the functionality of the structure.

It is about five decades that vibration based health monitoring of structures is utilized to evaluate the conditions of the structures. At the beginning, it was basically focused over the offshore oil industries and aerospace engineering problems and now it is widespread throughout the civil and mechanical engineering communities as well. In this context, the damage can be identified as the change in: the boundary conditions of the structure, the geometrical/material properties of the composing elements and or the connectivity of these elements.

Damage identification methods are the main component of structural health monitoring which process the data in order to detect damages in the structure. Damage detection of the structures can be categorized into 4 groups based on their level of identification: I) identifying the damage existence, II) identifying the geometrical location of the damage, III) quantification of the severity of damage and IV) evaluation of the remaining service life of the structure. Most of the literature is concentrated on the level 1 to 3 of the detection on the controlled laboratory structures and some on-field tests.

Several extensive review papers can be found in literature on the topic of damage detection e.g. ([1] and [2]). Among the damage identification methods, the statistical

${ }_{1}^{1}$ Department of Civil Engineering, University of British Columbia, Vancouver, Canada

2 Inria, I4S, Campus de Beaulieu, Rennes, France

3 IFSTTAR, COSYS, SII, Bouguenais, France 
subspace damage detection method has a strong theoretical background which showed to be robust in practice for detecting the damage in S101 bridge [3]. Several studies were also carried out on this technique for detecting the damage in [4]-[7]. In this paper the statistical subspace damage localization (SSDL) approach [8], [9] is employed in localizing the damage in a real structure, namely the Yellow frame. This method uses vibration measurements of the structure in a (healthy) reference state and in the damaged state, as well as a finite element (FE) model in the reference state. Damage is localized through statistical tests on the parameters of the model, avoiding the FE updating problem. Several methodologies and theories are developed and demonstrated in order to enable the practical implementation of this technique, which are shown in this paper.

First, the SSDL technique will be reviewed and then the methods and theories will be proposed. At the end the results of these method on the Yellow frame will be shown. Following that, the conclusions and discussions are presented.

\section{STATISTICAL SUBSPACE DAMAGE LOCALIZATION TECHNIQUE}

In this section, the theoretical background of the statistical subspace damage localization technique is introduced, mainly based on references [8], [9]. This technique can detect the damage in a structure by creating a subspace from measurement data in a reference (healthy) state. This subspace is based on the modes of the structure but without actual computation of them. This subspace is employed in a statistical comparison along with the data measured from the possibly damaged structure in assessing the condition of the structure. In this statistical comparison, sensitivities from an analytical model of the structure in the reference state are used, without computation of the modes from the test data.

\section{Output-Only Covariance Based Subspace System Identification}

The discrete-time state-space representation of the dynamic equilibrium equation of a model can be written by performing sampling with time step $\tau$ in step $k$ as

$$
\left\{\begin{array}{c}
x_{k+1}=F x_{k}+w_{k} \\
y_{k}=H x_{k}+\varepsilon_{k}
\end{array}\right.
$$

in which $x \in \mathbb{R}^{n}$ is representing the state of the system and the measured output is represented by $y \in \mathbb{R}^{r}$. Parameter $n$ is the system order and $r$ is the number of sensors. $F \in \mathbb{R}^{n \times n}$ is the state transition matrix and $H \in \mathbb{R}^{r \times n}$ is the observation matrix. The state noise $w_{k}$ and output measurement noise $\varepsilon_{k}$ are generally assumed to be white noise for output-only system identification approaches.

By defining the output covariance as $R_{i}=\mathbf{E}\left(y_{k} y_{k-i}^{T}\right)$, the block Hankel matrix $\mathbf{H}_{p+1, q}$ is composed as

$$
\mathbf{H}_{p+1, q}=\left[\begin{array}{cccc}
R_{1} & R_{2} & \cdots & R_{q} \\
R_{2} & R_{3} & \cdots & R_{p+1} \\
\vdots & \vdots & \ddots & \vdots \\
R_{p+1} & R_{p+2} & \cdots & R_{p+q}
\end{array}\right]=\operatorname{Hank}\left(R_{i}\right)
$$


Matrix $\mathbf{H}_{p+1, q}$ contains information from the dynamic properties of the system and, hence, any change in the system will be reflected in a change in $\mathbf{H}_{p+1, q}$. A simple way of monitoring those changes is to compare $\mathbf{H}_{p+1, q}$ to its value in the reference state or even simply to compute the cross product of it and its left null-space at the reference state $S_{0}$. Then, if there is no change in the system

$$
S_{0}^{T} \mathbf{H}_{p+1, q}=0
$$

and if there is a change in the system $S_{0}^{T} \mathbf{H}_{p+1, q} \neq 0$. Due to the stochastic nature of data, such a small change from zero is additionally corrupted by noise modeled as a random normally distributed variable, namely the residual function $\zeta$, defined as

$$
\zeta=\sqrt{N} \operatorname{vec}\left(S_{0}^{T} \hat{\mathbf{H}}_{p+1, q}\right)
$$

where $N$ represents the number of samples used in the computation of $\hat{\mathbf{H}}_{p+1, q}$ estimated from $R_{i}=\frac{1}{N} \sum_{k=1}^{N} y_{k} y_{k-i}^{T}$. By defining the system parameter $\theta$ as the current physical properties of the structure such as the stiffness of each element, and $\theta_{0}$ as the physical properties of the reference structure (undamaged), we have

$$
\theta=\theta_{0}+\delta \theta / \sqrt{N}
$$

where $\delta \theta=\sqrt{N}\left(\theta-\theta_{0}\right)$ is defined as the (unknown) normalized parameter change vector. Therefore, by defining two hypotheses based on the condition of the structure as

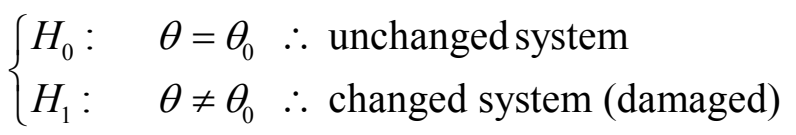

the defined residual can be proved to be asymptotically normally distributed (for large $N)$ with

$$
\zeta \rightarrow \begin{cases}\mathcal{N}(0, \Sigma) & \text { under } H_{0} \\ \mathcal{N}(J \delta \theta, \Sigma) & \text { under } H_{1}\end{cases}
$$

in which $J$ represents the asymptotic sensitivity of the residual with respect to the system parameterization, and $\Sigma$ is the covariance matrix of the residual.

\section{Parametric Hypothesis Test}

In order to decide between hypotheses (6), a generalized likelihood ratio (GLR) test is employed [8] which results in the following $\chi^{2}$-value, representing a damage index:

$$
\chi^{2}=\zeta^{T} \Sigma^{-1} J\left(J^{T} \Sigma^{-1} J\right)^{-1} J^{T} \Sigma^{-1} \zeta
$$

If this value exceeds a threshold it indicates the existence of damage in the system. In order to locate the damage two procedures, i.e. MinMax and sensitivity based approaches, can be employed. While in the sensitivity based approach, (8) is used by 
simply defining $J=J_{p_{k}}$ where $J_{p_{k}}$ is a vector of Jacobians corresponding to the parameter $p_{k}$, in the MinMax approach a robust $\chi^{2}$-value is defined as

$$
\chi^{* 2}\left(p_{k}\right)=\zeta_{k}^{* T} F_{k}^{*-1} \zeta_{k}^{*}
$$

where $F_{k}^{*}$ is a part of a Fisher-information-matrix for parameter $p_{k}$ while removing (projecting out) the effect of other parameters [9]. Similarly, the robust residual $\zeta_{k}^{*}$ is defined. The sensitivity approach should be less effective than the MinMax approach in case of a highly dependent parameterization. Herein, these two approaches are employed in localizing the damage for the Yellow frame.

\section{JACOBIAN COMPUTATION}

The residual defined in the SSDL method is derived from operations on the measured data and has a statistical background while the FE model is directly connected to the physical behaviour of the structure. A relation linking the data-based residual to the physical model is needed, which is the sensitivity of the residual with respect to the physical parameterization. This link is made by the derivative chain in (10). This will let us benefit from a physical model of the structure along with the measured data to localize the damage. Assume for simplicity that each element of the structure is uniquely related to one physical parameter $p_{k}$. The chain rule is employed in connecting the residual function (7) to the physical parameters $p_{k}$ as

$$
J\left(p_{k}\right)=J(\lambda, \varphi) J_{(\mu, \psi)}^{(\lambda, \varphi)} J_{(f, \xi, \wp)}^{(\mu, \psi)} J_{p_{k}}^{(f, \xi, \wp)}
$$

in which $(\lambda, \varphi)$ and $(\mu, \psi)$ represent, in order, the discrete and continuous time eigenstructure. The modal parameters, i.e. natural frequencies, mode shapes and damping values, are denoted by $(f, \xi, \wp) . J(\lambda, \varphi)$ is the sensitivity of the residual to the eigenstructure $(\lambda, \varphi)$ and $J_{\circ}^{\bullet}$ is the consistent Jacobian of parameters $\bullet$ with respect to $\circ$.

For the computation of $J\left(p_{k}\right)$, there should be a decision made on the use of the estimated eigenstructure from the measured data versus the modal parameters from the analytical model, on each term in the computation of Jacobians. The parameters estimated from the measured data are assumed to be more precise when the identification is well performed. In general, the model updating on the analytical model is not necessary and not needed in the SSDL procedure unless the modal parameters are very different from the identified ones and the model is not a good representative of the dynamic behaviour of the structure. The important accuracy needed for the damage localization is in the sensitivity values of modal parameters with respect to physical parameters. Herein, two options are described and their performance will be investigated in the case study.

Configuration 1: All terms in (10) are evaluated from the analytical model. In this way, all the parameters are consistent and the Jacobian is purely computed from the analytical model. The modal parameters of the analytical model are used for the 
computation of $J(\lambda, \varphi)$, and only the Hankel matrix $\mathbf{H}_{p+1, q}$ and $S_{0}$ are computed from the measured data.

Configuration 2: The first, second and third terms of the Jacobian are evaluated from the eigenstructure estimated from the measured data and the last term is evaluated from the modal parameters of the analytical model as in [8]. It should be noted that the modal parameters of the analytical model and the eigenstructure of the measured data need to be identified and adjusted in terms of scaling and order. Hence, one system identification is needed in the reference state prior to the localization of damage.

After the computation of Jacobians the vectors corresponding to each elements need to be clustered if they are close, using the Hierarchical Fisher-information-matrix-based clustering (HFC) approach [10]. This clustering is based on the the statistical properties of the data as well as the modal behaviour of the elements that in turn is related to their geometrical and physical closeness and modal direction in the considered mode shapes.

\section{SCALING OF MODE SHAPES}

By using the second configuration described in previous subsection, there is a need in scaling the mode shapes obtained from the analytical model, i.e. $\wp$, and measured data, i.e. $\varphi$ and $\psi$. This scaling can be performed in typical ways such as modal mass scaling or unit maximum member. However, usually the mass matrix of the analytical model is complicated to truncate and the mode shapes are obtained from limited number of degrees of freedoms on which a sensor is located. Therefore, the general scaling of these vectors is performed as follows. Since the scaling of the mode shapes is not matching,

$$
\alpha \wp_{j}=\varphi_{j}
$$

where $\alpha$ is a constant scalar. Thus, the adjusted mode shapes $\tilde{\wp}$, can be evaluated as

$$
\tilde{\wp}_{j}=\frac{\wp_{j}^{H} \varphi_{j}}{\wp_{j}^{H} \wp_{j}} \wp_{j}
$$

\section{Coupled Mode Shapes Scaling}

Coupled mode shapes happen mostly in symmetric or nearly symmetric structures which have sets of two close eigenvalues. In these cases, the identified mode shapes are mixed and result in a linear combination of the analytical mode shapes. In order to solve this issue, the scaling needs to be done by solving a double linear equation. Let $\wp_{1}$ and $\wp_{2}$ be the two coupled mode shapes corresponding to the analytical mode shapes $\varphi_{1}$ and $\varphi_{2}$, respectively. The linear combination is written as

$$
C \Gamma_{1,2}=\Phi_{1,2} \text { where } C=\left[\begin{array}{ll}
c_{1} & c_{2} \\
c_{3} & c_{4}
\end{array}\right], \Gamma_{1,2}=\left[\begin{array}{c}
\wp_{1}^{T} \\
\wp_{2}^{T}
\end{array}\right] \text { and } \Phi_{1,2}=\left[\begin{array}{c}
\varphi_{1}^{T} \\
\varphi_{2}^{T}
\end{array}\right]
$$


Therefore, we have $C=\Phi_{1,2} \Gamma_{1,2}^{\dagger}$ and then the scaled and decoupled mode shapes $\tilde{\Gamma}_{1,2}=\left[\begin{array}{c}\tilde{\wp}_{1}^{T} \\ \tilde{\wp}_{2}^{T}\end{array}\right]$ can be evaluated as

$$
\tilde{\Gamma}_{1,2}=\Phi_{1,2} \Gamma_{1,2}^{\dagger} \Gamma_{1,2}
$$

\section{CASE STUDY: THE YELLOW FRAME}

The Yellow frame is a modular 4 story, scaled (1/3) steel frame established in 2016 at the University of British Columbia (UBC), shown in Figure 1. Several damage scenarios are designed and tested by removal of braces of the structure to test the SSDL method. This structure is $3.6 \mathrm{~m}$ high and is composed of 2 spans in each direction with the total length of $2.5 \mathrm{~m}$. Each floor of the structure is carrying dead loads applied to the structure by using 4 steel plates distributed on each level. Based on Figure 1.c, the considered damage scenarios are defined as Table 1.

The FE model of this structure is built and the sensitivity analysis of the mode shapes and natural frequencies with respect to each brace is computed using a finite difference approach. Subsequently, using the proposed scaling/decoupling approach, the coupled mode shapes can be perfectly decoupled; this is demonstrated in the following figure for mode shapes 1 and 2 which are among the coupled mode shapes.

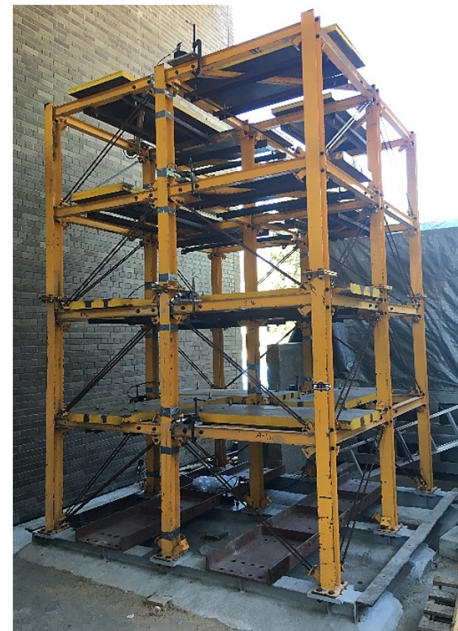

(a)

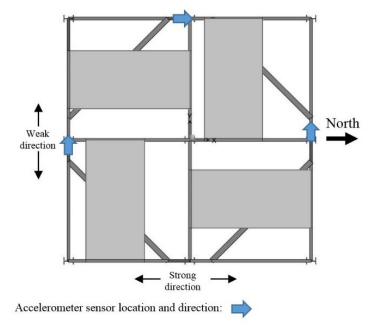

(b)

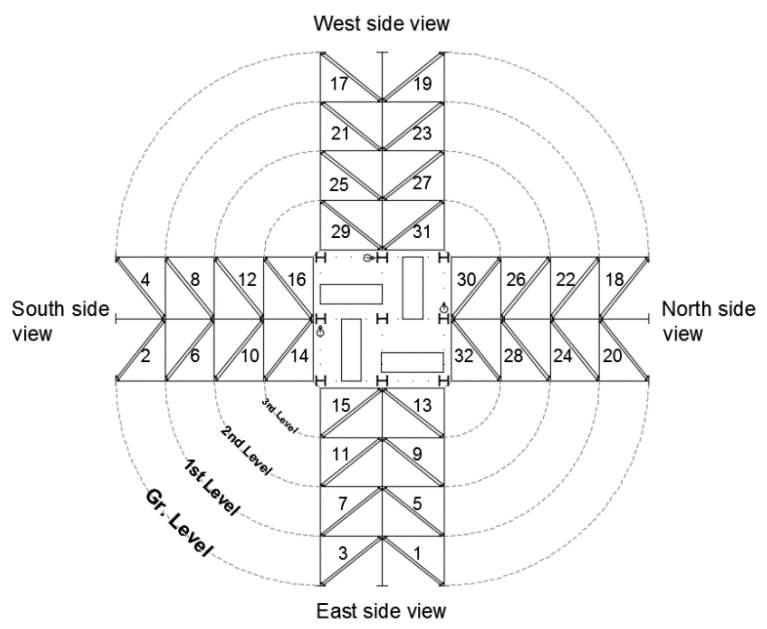

(c)

Figure 1. (a) The Yellow frame structure, (b) the schematic plan of the structure showing the location of sensors, (c) the numbering of the braces of the structure 
TABLE I. DAMAGE SCENARIOS OF THE YELLOW FRAME

\begin{tabular}{|cc|}
\hline $\begin{array}{c}\text { Scenario } \\
\text { number }\end{array}$ & $\begin{array}{c}\text { Removed braces (number } \\
\text { of braces removed) }\end{array}$ \\
\hline S1 & 2 (II), 4 (II), 18 (II), 20 (II) \\
\hline S2 & 21 (II), 23 (II) \\
\hline
\end{tabular}
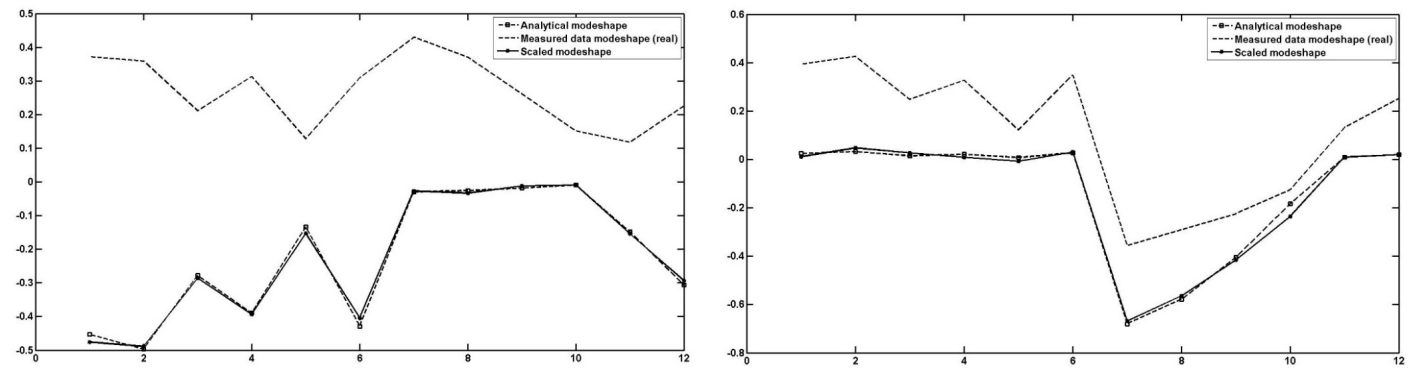

Figure 2. Mode shapes decoupling and scaling; (left: $1^{\text {st }}$, right: $2^{\text {nd }}$ mode shape)

The sensitivities are used in composing the Jacobians either from configuration 1 or 2. Using the HFC approach the elements are clustered based on the Jacobian columns. Finally the $\chi^{2}$-test is computed for each element (brace) using the sensitivity and MinMax approaches as illustrated in Figure 3. From these results it can be seen that the damage localization works best with Jacobian configuration 1 using the MinMax approach. With this setting, the test reacts well on the damaged elements in both scenarios S1 and S2, localizing the damage correctly. For the multiple distinct damage scenario S1, the MinMax approach using Jacobian configuration 1 seems to be the only robust damage localization solution. For the simpler case, where damage occurs only at a pair of neighboring elements, damage can also be localized by MinMax approach in configuration 2 , whereas the sensitivity approach only works in configuration 1 .
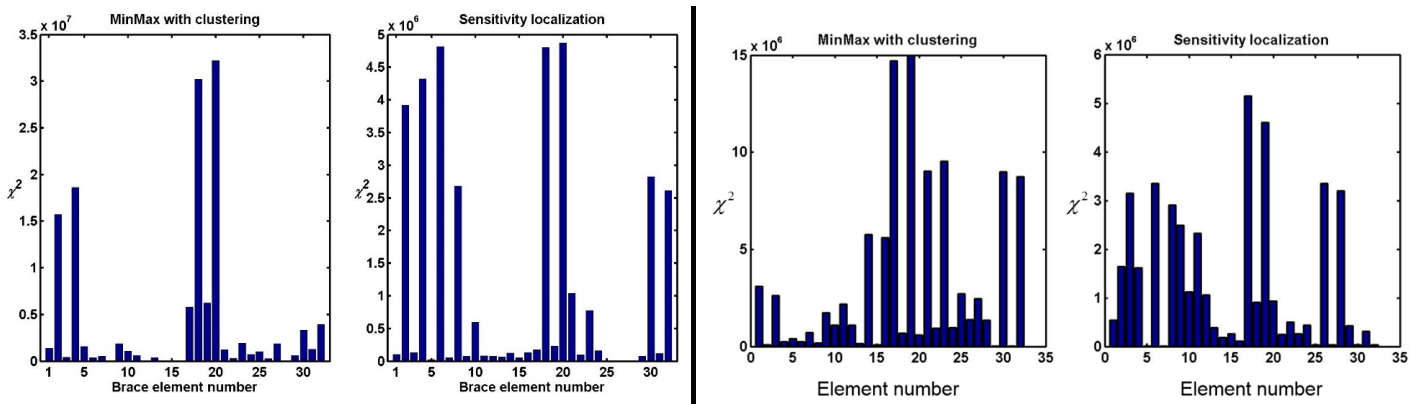

Scenario S1 with configuration 1
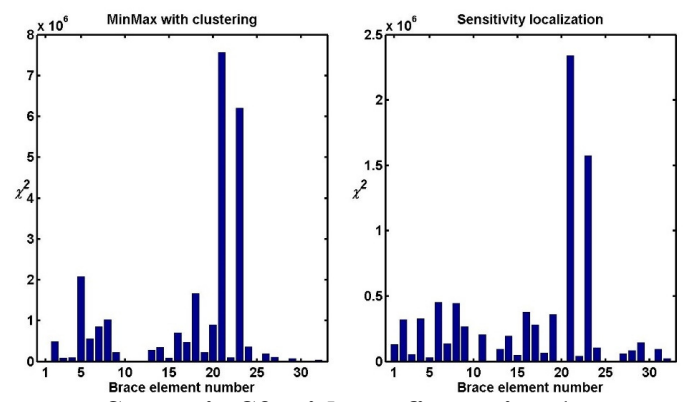

Scenario $\mathbf{S 2}$ with configuration 1

Scenario S1 with configuration 2
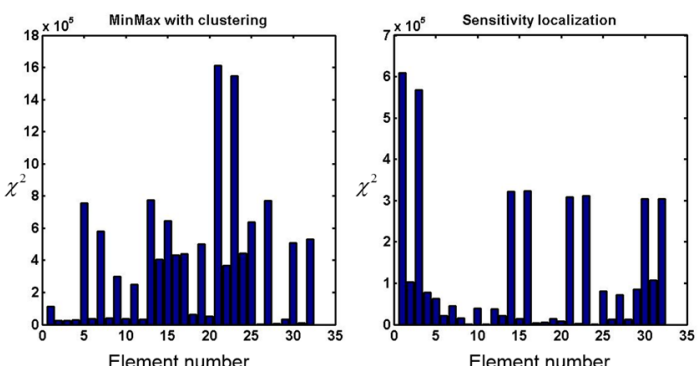

Scenario $\mathbf{S 2}$ with configuration 2

Figure 3. SSDL method from sensitivity based and MinMax approaches for two configuration schemes of the Jacobian computation 


\section{DISCUSSION AND CONCLUSIONS}

In this paper, the experimental data measured from the Yellow frame test was employed in validating the proposed methods allowing the SSDL approach to localize the damage in practice. It was shown that the SSDL method can localize the damage in this structure effectively for two damage scenarios.

Two configurations of the Jacobian matrix were investigated. It was illustrated that using the modes from the analytical model in composing the Jacobian matrix results in a more robust damage identification. The reasoning behind this choice relies to the following requirement: in order to compose the Jacobians from analytical and real data modal parameters, modes need to be matched from data and FE model; In that particular application, the number of identified parameters came out to be less than the analytical modal parameters ( 8 versus 10$)$. This resulted in less precision in the tests using Jacobians derived from Configuration 2, where the estimates were used.

The MinMax and sensitivity based approach were used in localizing the damage. The MinMax approach requires a pre-clustering of the parameterization to be effective and the clustering obtained from HFC appeared to be an appropriate clustering scheme for the proposed method. Finally, it was observed that the MinMax test using Jacobians derived using Configuration 1 based on the analytical modes is the best choice to localize the damage for both simple and multiple distinct damages.

\section{REFERENCES}

[1] W. Fan and P. Qiao, "Vibration-based Damage Identification Methods: A Review and Comparative Study," Struct. Heal. Monit., vol. 10, no. 1, pp. 83-111, Apr. 2010.

[2] S. W. Doebling, C. R. Farrar, and M. B. Prime, "A Summary Review of Vibration-Based Damage Identification Methods," Shock Vib. Dig., vol. 30, no. 2, pp. 91-105, 1998.

[3] M. Döhler, F. Hille, L. Mevel, and W. Rücker, "Structural health monitoring with statistical methods during progressive damage test of S101 Bridge," Eng. Struct., vol. 69, pp. 183-193, Jun. 2014.

[4] S. Allahdadian, C. Ventura, P. Andersen, L. Mevel, and M. Döhler, "Investigation on the sensitivity of subspace based damage detection technique to damage and noise levels," in IOMAC-International Operational Modal Analysis Conference, 2015.

[5] S. Allahdadian, C. E. Ventura, P. Andersen, L. Mevel, and M. Döhler, "Sensitivity Evaluation of Subspace-Based Damage Detection Method to Different Types of Damage," Springer, Cham, 2015, pp. 11-18.

[6] S. Allahdadian, C. Ventura, P. Andersen, L. Mevel, and M. Döhler, "Subspace based damage detection technique: investigation on the effect of number of samples," in CCEE-11th Canadian Conference on Earthquake Engineering, 2015.

[7] S. Allahdadian, M. Döhler, C. E. Ventura, and L. Mevel, "On the Influence of Sample Length and Measurement Noise on the Stochastic Subspace Damage Detection Technique," Springer, Cham, 2016, pp. 35-46.

[8] E. Balmès, M. Basseville, L. Mevel, H. Nasser, and W. Zhou, "Statistical model-based damage localization: A combined subspace-based and substructuring approach," Struct. Control Heal. Monit., vol. 15, no. 6, pp. 857-875, 2008.

[9] M. Döhler, L. Mevel, and Q. Zhang, "Fault detection, isolation and quantification from Gaussian residuals with application to structural damage diagnosis," Annu. Rev. Control, vol. 42, pp. 244 256, 2016.

[10] Saeid Allahdadian, "Practical Damage Identification of Structures Using the Statistical Subspace Damage Detection Technique," University of British Columbia, 2017. 